\title{
PERIPROCEDURAL MYOCARDIAL INFARCTION
}

\section{Tripti Deb}

The First Global MI Task Force in 2000 implied that any necrosis in the setting of myocardial ischaemia should be labelled as MI. The development of more sensitive assays for markers of myocardial necrosis mandates further revision, particularly when such necrosis occurs in the setting of the critically ill, after percutaneous coronary procedures or after cardiac surgery. The Third Global MI Task Force by Thygesen et al in 2012 recognizes that very small amounts of myocardial injury or necrosis can be detected by biochemical markers and/or imaging.

Peri-procedural myocardial injury or infarction (Type 4 and 5) may occur at some stages in the instrumentation of the heart that is required during mechanical revascularization procedures, either by $\mathrm{PCI}$ or by coronary artery bypass grafting (CABG). Elevated cTn values may be detected following these procedures, since various insults may occur that can lead to myocardial injury with necrosis. It is likely that limitation of such injury is beneficial to the patient: however, a threshold for a worsening prognosis, related to an asymptomatic increase of cardiac biomarker values in the absence of procedural complications, is not well defined. Subcategories of PCI-related MI are connected to stent thrombosis and restenosis that may happen after the primary procedure.

Blood samples for the measurement of cTn should be drawn on first assessment and repeated 3-6 h later. Later samples are required if further ischaemic episodes occur, or when the timing of the initial symptoms is unclear.

To establish the diagnosis of MI, a rise and/or fall in values with at least one value above the decision level is required, coupled with a strong pre-test likelihood. The demonstration of a rising and/or falling pattern is needed to distinguish acute-from chronic elevations in cTn concentrations that are associated with structural heart disease. For example, patients with renal failure or HF can have significant chronic elevations in cTn. These elevations can be marked, as seen in many patients with MI, but do not change acutely. $\underline{z}$ However, a rising or falling pattern is not absolutely necessary to make the diagnosis of MI if a patient with a high pre-test risk of MI presents late after symptom onset; for example, near the peak of the cTn time-concentration curve or on the slow-declining portion of that curve, when detecting a changing pattern can be problematic. Values may remain elevated for 2 weeks or more following the onset of myocyte necrosis.

B M Harris et al in 2000 measured CK-MB, cTnT and cardiac troponin I (cTnI) to ascertain which is the most sensitive marker for minor myocardial damage. Blood samples were taken both before and 6, 14 and $24 \mathrm{~h}$ after the procedure in 109 patients ( 77 men) with angina, 42 of whom had unstable angina. Of the 109 patients, 86 had a stent inserted (21 as a primary stent), nine had PTCA, eight had RA and six intracoronary brachytherapy and concluded that CTnI was the most sensitive indicator of minor myocardial damage, but at the recommended cutoff of $0.1 \mathrm{mcg} / \mathrm{L}$ may be overly sensitive.

The present study observed that Peri-procedural myocardial infarction (PMI) is a frequent complication of percutaneous coronary intervention and investigated the incidence and clinical outcomes of peri-procedural MI patients over one year follow up.

\section{REFERENCES:}

1. Myocardial infarction redefined-A consensus document of the Joint European Society of Cardiology/American College of Cardiology Committee for the redefinition of myocardial infarction J Am CollCardiol 2000;36:959-69

2. Kristian Thygesen, Joseph S. Alpert, Allan S. Jaffe, et al. Third Universal Definition of Myocardial Infarction ESC Committee for Practice Guidelines (CPG) J Am Coll; 2012, 60(16), 1581-1598. 
3. Harris BM, Nageh T, Marsden JT, Thomas MR, Sherwood RA. Comparison of cardiac troponin $\mathrm{T}$ and $\mathrm{I}$ and CK-MB for the detection of minor myocardial damage during interventional cardiac procedures. Ann ClinBiochem 2000;37:764-9.

4. Jaffe AS. Chasing troponin: how low can you go if you can see the rise? J Am CollCardiol 2006;48:1763-4.
5. Thygesen K, Mair J, Katus H, et al., Study Group on Biomarkers in Cardiology of the ESC Working Group on Acute Cardiac Care. Recommendations for the use of cardiac troponin measurement in acute cardiac care. Eur Heart J 2010;31:2197-204. 